\title{
SQUARE INTEGRABLE REPRESENTATIONS OF SEMISIMPLE LIE GROUPS $\left({ }^{1}\right)$
}

\author{
BY
}

JUAN A. TIRAO

\begin{abstract}
Let $D$ be a bounded symmetric domain. Let $G$ be the universal covering group of the identity component $A_{0}(D)$ of the group of all holomorphic diffeomorphisms of $D$ onto itself. In this case, any $G$-homogeneous vector bundle $E \rightarrow D$ admits a natural structure of $G$-homogeneous holomorphic vector bundles. The vector bundle $E \rightarrow D$ must be holomorphically trivial, since $D$ is a Stein manifold. We exhibit explicitly a holomorphic trivialization of $E-$ $D$ by defining a map $\Phi: G \rightarrow G L(V)$ ( $V$ being the fiber of the vector bundle) which extends the classical "universal factor of automorphy" for the action of $A_{0}(D)$ on $D$. Then, we study the space $H$ of all square integrable holomorphic sections of $E-D$. The natural action of $G$ on $H$ defines a unitary irreducible representation of $G$. The representations obtained in this way are square inte. grable over $G / Z$ ( $Z$ denotes the center of $G$ ) in the sense that the absolute values of their matrix coefficients are in $L_{2}(G / Z)$.
\end{abstract}

1. Introduction. Now, let $G$ be any connected covering group of $A_{0}(D)$. Choose a base point $\circ \in D$ and represent $D=G / K$.

When $G$ admits a finite dimensional faithful representation (in which case $G$ has infinite fundamental group), M. S. Narasimhan and K. Okamoto [9] have shown that most of the discrete classes of $G$ (obtained by Harish-Chandra in [6]) can be realized on spaces of square integrable harmonic forms of type $(0, q)$ with coefficients in homogene ous holomorphic vector bundles on $G / K$ arising from finite dimensional irreducible unitary representations of $K$.

If we assume that $G$ has a finite dimensional faithful representation we can consider a complex form $G_{c}$ of $G$ and realize $G / K$ as an open $G$-orbit in a complex manifold $G_{c} / L$ where $L=K_{c} P_{+}$(see $\$ 2$ ) is a closed complex Lie subgroup of $G_{c}$. Homogeneous vector bundles are obtained as associated bundles $E_{\tau} \rightarrow D$ to the principal $K$-bundle $G \rightarrow D$ where $r$ is a continuous representation of $K$ on a finite dimensional complex vector space $V$. If $r$ denotes an irreducible unitary

Received by the editors June 12, 1972.

AMS (MOS) subject classifications (1970). Primary 22E45; Secondary 32M15.

Key words and phrases. Bounded symmetric domains, homogeneous holomorphic vector bundles, square integrable holomorphic sections.

( ${ }^{1}$ ) This research was partially supported by a fellowship from the Consejo Nacional de Investigaciones Cientificas y Técnicas de Argentina.

Copyright $\odot$ 1974, American Mathematical Society 
representation of $K$, then $r$ is uniquely extended to a holomorphic representation $\hat{\tau}^{\prime}$ of $L$ which is trivial on $P_{+}$. Then the homogeneous vector bundle $E_{\tau} \rightarrow G / K$ is the restriction of the homogeneous holomorphic vector bundle $E_{\gamma^{\circ}} \rightarrow G_{c} / L$ to the open submanifold $G / K$ of $G_{c} / L$. In this way $E_{\tau} \rightarrow G / K$ picks up a structure of a homogeneous holomorphic vector bundle.

When we drop the requirement that $G$ have a complex form, we are led to consider the following more general problem.

The existence and un iqueness of homogeneous bolomorphic vector bundle structures for homogeneous vector bundles over complex manifolds bomogeneous under a Lie group G. This is solved in [10].

In $\$ 2$ we apply these results to the case when $D$ is a bounded symmetric domain and $G$ is the universal covering group of $A_{0}(D)$. In this case, the homogeneous vector bundle $E_{\tau} \rightarrow D$ admits a natural structure of $G$-homogeneous holomorphic vector bundle which is described in Theorem 2.1. In the second part of $\$ 2$ we are concerned with the problem of constructing explicitly a holomorphic trivialization of $E_{\tau} \rightarrow D$. This is achieved by defining a map $\Phi: G \rightarrow G l(V)$ with the properties stated in The orem 2.3. Because of this, one is in good position to study the space $H$ of all square integrable holomorphic sections $E_{\tau} \rightarrow D$ (whenever $\tau$ is a unitary irreducible representation of $K$ ). The natural action of $G$ on $H$ defines a unitary irreducible representation of $G$ (cf. Theorem 3.1). The representations obtained in this way are unitarily equivalent to those studied in [3] and [4]. These representations are square integrable over $G / Z$, where $Z$ denotes the center of $G$.

An application of our constructions to the case where $G$ is a connected covering group of $A_{0}(D)$ implies that the Narasimhan-Okamoto result now extends without change to the case where $G$ has finite center. The extension to an arbitrary $G$ depends only on the generalization of the Harish-Chandra character the ory of the discrete series representations, [5], [6] to that of square integrable ones.

The material of this paper is contained in the author's doctoral dissertation presented to the University of California, Berkeley. The author wishes to thank Professor Joseph A. Wolf for much helpful advice and patient encouragement in the preparation of this dissertation.

2. Homogeneous holomorphic vector bundles over bounded symmetric domains. A bounded domain is a bounded, open connected subset of the complex vector space $C^{N}, N$ being a positive integer. A bounded domain $D$ is called symmetric if each $z \in D$ is an isolated fixed point of an involutive holomorphic diffeomorphism $s_{z}$ of $D$ onto itself. If such a diffeomorphism $s_{z}$ exists it is unique. Let $G$ be a connected covering group of the identity component $A_{0}(D)$ of the group of all holomorphic diffeomorphisms of $D$ onto itself. Then $G$ is transitive on $D$. 
Choose a base point $o \in D$ and represent $D=G / K$. Let $\sigma$ denote the automorphism $g \rightarrow s_{0} g s_{0}$ of $A_{0}(D)$ and let $g_{0}$ denote the Lie algebra of $A_{0}(D)$. If we put $\dot{\sigma}=$ $d \sigma_{e}$ we have the direct decomposition

$$
g_{0}=E_{0}+p_{0}
$$

where $E_{0}$ coincides with the Lie algebra of $K$ and the +1 eigenspace of $\dot{\sigma}$ and $p_{0}$ is the -1 eigenspace of $\dot{\sigma}$. Let $g, k, p$ denote the complexifications of $g_{0}$, $E_{0}$, and $p_{0}$ respectively. We denote by $\pi$ the canonical projection mapping $G$ onto $D$. We may identify $p_{0}$ with the real tangent space $D_{0}$ of $D$ at the point 0 by the mapping $Y \rightarrow d \pi_{e} Y_{e}\left(Y \in F_{0}\right)$. We denote by $T_{z}$ (resp. $\bar{T}_{z}$ ) the holomorphic (resp. the antiholomorphic) tangent space of $D$ at $z$. Put

$$
p_{-}=\left\{Y \in p_{0}: d \pi_{e} Y_{e} \in T_{0}\right\}, \quad p_{+}=\left\{Y \in p: d \pi_{e} Y_{e} \in \bar{T}_{0}\right\}
$$

As a corollary of Theorem 3.6 of [10] we have the following result:

2.1. Theorem. Let $\tau$ be a continuous representation of $K$ on a finite dimen-sional vector space, $E_{\tau} \rightarrow D$ the associated G-bomogeneous complex vector bundle. Then $E_{\tau} \rightarrow D$ has a natural structure of G-homogeneous bolomorphic vector bundle for which the bolomorphic sections $s$ over an open set $U \subset D$ are characterized by

section: $X f_{s}+\tau(X) f_{s}=0$ for all $X \in E_{0}$,

bolomorphic: $X f_{s}=0$ for all $X \in p_{+}$.

Proof. In [10] we have defined a complex subalgebra $\mathfrak{l}$ of $g$ by

$$
\zeta=\left\{X \in g: X_{0}=0\right\} \text {. }
$$

From the definitions it follows easily that $l=E+p_{+}$. Since $l$ is invariant under the complex linear extension of $\dot{\sigma}$ it follows that $p_{+}=\mathfrak{l} \cap p$ and therefore $\left[\mathcal{k}, p_{+}\right]$ $\subset[E, p] \cap \mathfrak{l} \subset p \cap \mathfrak{l}=p_{+}$. Now we want to prove that $\mathfrak{p}_{+}$is an abelian ideal of $\mathfrak{l}$. Let $J_{0}$ denote the complex structure of $D_{0}$ and let $E_{0}^{*}$ denote the Lie algebra of the linear isotropy group $K^{*}$ corresponding to $K$. Then $J_{0}$ belongs to the center $c_{0}$ of $\mathfrak{E}_{0}^{*}$ (see [7]). Identifying the Lie algebras $\mathcal{E}_{0}$ and $\mathfrak{E}_{0}^{*}$, for $X, Y \in p_{+}$we have

$$
0=\left[J_{0},[X, Y]\right]=\left[\left[J_{0}, X\right], Y\right]+\left[X,\left[J_{0}, Y\right]\right]=-2 i[X, Y]
$$

since $\left[p_{,}, p\right] \subset \ell$. This shows that $\left[p_{+}, p_{+}\right]=0$.

Since $K$ is connected there is a unique extension $\lambda$ of $\tau$ from $K$ to $\mathfrak{l}$ such that $\lambda \mid p_{+}=0$. Now Theorem 3.6 of $[10]$ provides the required $G$-homogeneous holomorphic structure for $E_{\tau} \rightarrow D$. This completes the proof of the theorem. 
Let $M$ be a connected complex manifold with a Hermitian structure; $M$ is said to be a Hermitian symmetric space if each point $p \in M$ is an isolated fixed point of an involutive holomorphic isometry $s_{p}$ of $M$.

For any bounded domain $D$ the Bergman kernel function of $D$ defines a Kählerian structure on $D$, i.e. a Hermitian structure such that the almost complex structure $J$ of $D$ is invariant under parallelism. With respect to this structure any holomorphic diffeomorphism of $D$ onto itself is an isometry, therefore any bounded symmetric domain is a Hermitian symmetric space. Moreover, $D$ is a Hermitian symmetric space of noncompact type, which means that the Lie algebra $g_{0}$ of $A_{0}(D)$ is semisimple without compact factors and that $(2.1)$ is a Cartan decomposition of $g_{0}$.

Conversely, if $M$ is a Hermitian symmetric space of noncompact type, then there exist a bounded symmetric domain $D$ and a holomorphic diffeomorphism of $M$ onto $D$. We need to recall the Harish-Chandra realization of $M$ as a bounded symmetric domain $D$ in order to exhibit explicitly a holomorphic trivialization of $E_{\tau} \rightarrow D$. See [ 7] for details.

As before, let $A_{0}(M)$ be the identity component of the group of all isometries of $M$. Let $g_{0}$ denote its Lie algebra and let

$$
g_{0}=E_{0}+p_{0}
$$

be the Cartan decomposition of $g_{0}$ which arises from the symmetry with respect to some point $o$ in $M$. Let $c_{0}$ be the center of $\epsilon_{0}$ and let $\mathscr{G}_{0}$ be some maximal abelian subalgebra of $E_{0}$. Then $c_{0} \subset \mathscr{G}_{0}$ and $\mathscr{G}_{0}$ is a maximal abelian subalgebra of $g_{0}$.

Let $g$ be the complexification of $g_{0}$ and let $c, \xi, f, f$ be the complex subspaces of $g$ spanned by $c_{0}, \mathscr{G}_{0}, E_{0}, \mathfrak{F}_{0}$. Now $\mathfrak{G}$ is a Cartan subalgebra of $g$. Let $\Delta$ denote the set of nonzero roots of $g$ with respect to $\mathscr{G}$. Since $[G, \mathfrak{E}] \subset \mathfrak{E}$ and $[G, p] \subset p$, it is clear that either a root subspace $g^{a} \subset \mathcal{E}$ or $g^{a} \subset p(\alpha \in \Delta)$. A root $a \in \Delta$ is called compact or noncompact according to $g^{a} \subset t^{k}$ or $g^{a} \subset R$ Moreover, $\left[E, p_{+}\right] \subset p_{+}$(see (2.2)), therefore there exists a subset $P_{n} \subset \Delta$ such that

$$
p_{+}=\sum_{a \in P_{n}} g^{a}
$$

Since $p_{+}=\nu_{0}\left(p_{-}\right)$and $\nu_{0}\left(g^{a}\right)=g^{-a}(a \in \Delta)$ where $\nu_{0}$ denotes the conjugation of $g$ with respect to $g_{0}$, we see that $p_{-}=\Sigma_{a \in P_{n}} g^{-a}$.

We can introduce a linear ordering in the dual of the real vector space $i f_{0}$ such that the set $P$ of all positive roots contains $P_{n}$. We choose an ordering with this property and whenever necessary we will be referring to it. 
Let $G_{c}$ denote the simply connected Lie group with Lie algebra $g$. Let $P_{-}$, $P_{+}, K_{c}$ denote the analytic subgroups of $G_{c}$ corresponding to the subalgebras $P_{-}, P_{+}$, and $E$, respectively. ( $P_{-}$and $P_{+}$are abelian subspaces of $F_{3}$ cf. the proof of Theorem 2.1.) Let $G_{0}, \bar{K}_{0}$ denote the analytic subgroups of $G_{c}$ corresponding to $g_{0}$ and $E_{0}$, respectively. The mapping $(q, k, p) \rightarrow q k p$ is a holomorphic diffeomorphism of $P_{-} \times K_{c} \times P_{+}$onto an open submanifold of $G_{c}$, containing $G_{0}$. For $x \in G_{0}$, let $\zeta(x)$ denote the unique element in $P_{\text {_ }}$ such that $x \in \zeta(x) K_{c} P_{+} \cdot P_{-}$and $P_{+}$are simply connected abelian Lie groups; let log: $P_{-}$ $\rightarrow P_{-}$be the inverse of the exponential mapping. The mapping

$$
x K_{0} \rightarrow \log \zeta(x), \quad x \in G_{0},
$$

is a holomorphic diffeomorphism of $r_{0} / K_{0}$ onto a bounded domain $D$ in the complex vector space $P_{-}$.

From now on, $G$ will denote the universal covering group of $A_{0}(M)$, and $K$ will denote the isotropy subgroup of $G$ at $o \in M$. Then we can make the identification $M=G / K$. Let $g \rightarrow \bar{g}, g \in G$, be the covering bomomorphis $m \rightarrow G_{0}$. The mapping

$$
\psi: g K \rightarrow \log \zeta(\bar{g}), \quad g \in G,
$$

is, therefore, a holomorphic diffeomorphism of $G / K$ onto $D$.

Let $r$ be a representation of $K$ on the finite dimensional complex vector space $V$. We want to define a holomorphic equivalence between the holomorphic vector bundles $E_{\tau} \rightarrow G / K$ and the trivial vector bundle $D \times V \rightarrow D$. This amounts to defining a holomorphic diffeomorphism $\Psi$ such that the diagram

$$
\begin{aligned}
& G \times{ }_{\tau} V \stackrel{\boldsymbol{\Psi}}{\rightarrow} D \times V \\
& \begin{array}{cc}
\downarrow & \downarrow \\
G / K & \mathbb{P}
\end{array}
\end{aligned}
$$

commutes and induces linear isomorphisms between fibers. To define such a map $\Psi$ is equivalent to defining a map $\Phi: G \rightarrow \mathrm{Gl}(V)$ such that

$$
\Phi(g k)=\Phi(g) \tau(k) \text { for all } g \in G \text {; all } k \in K \text {; }
$$

$\Psi$ and $\Phi$ being related by

$$
\Psi[g, v]=(\psi(g K), \Phi(g) v), \quad g \in G, v \in V .
$$

To motivate our definition of the map $\Phi$ in the general case let us consider first the following example: The holomorphic tangent space of $G / K$ at $e K$ has been identified with $p_{-}$, and for every $k \in K$ the differential of the map $g K \rightarrow$ $k g K$ at $e K$ corresponds to the automorphism of $p_{-}$induced by $\operatorname{Ad}_{G}(k)$. This 
implies that the holomorphic tangent bundle of $G / K$ is the homogeneous vector bundle $E_{\tau}$, where $\tau$ denotes the representation of $K$ on $p_{-}$induced by $\mathrm{Ad}_{G}$. Let $\rho(g)$ denote the action of $G$ on $D$ given by

$$
\rho(g) \psi(x K)=\psi(g x K), \quad g, x \in G .
$$

Since the tangent bundle of $D$ is already presented as a trivial vector bundle we just have to compute the differential of $\rho(g)$ at the origin 0 .

2.2. Lemma (cf. Lemma 1.9 of [1]). Let $\kappa: G \rightarrow K_{c}$ be defined by $\bar{g} \epsilon$ $P_{-} \kappa(g) P_{+}$. Then

$$
d \rho(g)_{0}=\operatorname{Ad}_{G_{c}}(\kappa(g)) \mid p_{-} \text {for all } g \in G .
$$

Proof. Given $X \in p_{-}$, by definition we have

$$
\rho(g)(t X)=\log (\zeta(\bar{g} \exp t X)),
$$

for a sufficiently small $t$. Let $\bar{g}=q \kappa(g) p$ with $q \in P_{-}$and $p \in P_{+}$, then

$$
\bar{g} \exp t X=q \kappa(g) p \exp t X=q \kappa(g)(\exp t \operatorname{Ad}(p) X) p .
$$

Since $\left[p_{+}, p_{-}\right] \subset \notin,\left[\epsilon, p_{ \pm}\right] \subset p_{ \pm}$and $\left[p_{ \pm}, p_{ \pm}\right]=\{0\}$, for $Y \in p_{+}$we have

$$
\text { Ad }(\exp Y) X=X+[Y, X]+1 / 2[Y,[Y, X]] \text {. }
$$

Therefore we can write

$$
\operatorname{Ad}(p) X=X+Z \quad \text { with } Z \in \epsilon_{+} p_{+} .
$$

Putting these things together we obtain

$$
\begin{aligned}
d \rho(g)_{0} X & =[d / d t \log (\zeta(\bar{g} \exp t X))]_{t=0} \\
& =[d / d t \log (\zeta(q \kappa(g)(\exp t(X+Z)) p))]_{t=0} \\
& =\left[d / d t \log \left(\zeta\left(q(\exp t \operatorname{Ad}(\kappa(g)) X \exp t \operatorname{Ad}(\kappa(g)) Z)_{\kappa}(g) p\right)\right)\right]_{t=0} \\
& =[d / d t \log (q \exp t \operatorname{Ad}(\kappa(g)) X)]_{t=0}=\operatorname{Ad}(\kappa(g)) X,
\end{aligned}
$$

where we have used the fact that $K_{c} P_{+}$is a subgroup of $G_{c}$.

Lemma 2.2 tells us how we can define a holomorphic equivalence between the holomorphic tangent bundle $E_{\tau}$ of $G / K$ and the trivial bundle $D \times p_{-} \rightarrow D$. We can just take as the map $\Phi: G \rightarrow G l\left(p_{-}\right)$of (2.4) the map defined by

$$
\Phi(g)=\operatorname{Ad}_{G_{c}}(\kappa(g)) \mid p_{-}, \quad g \in G .
$$

The situation in this example is particularly simple because the representation $\tau$ of $K$ factors thru the restriction to $K_{0}$ of a representation of $K_{c}$. In the general case one has to be more careful. 
The Lie algebra $E_{0}$ being the Lie algebra of a compact Lie group, e.g. $K_{0}$, is the direct sum $E_{0}=C_{0}+\left[E_{0}, E_{0}\right]$ of its center and its derived algebra $E_{0}^{\prime}=$ $\left[E_{0}, E_{0}\right]$. If $K^{\prime}$ denotes the analytic subgroup of $K$ which corresponds to $\mathfrak{E}_{0}^{\prime}$, then $K^{\prime}$ is a compact, simply connected semisimple Lie group. Let $C$ be the analytic subgroup of $K$ with Lie algebra $c_{0}$. Then $C$ is a simply connected abelian Lie group and $K=C \times K^{\prime}$ (direct product). Let $E^{\prime}=[\mathrm{E}, \mathrm{t}]$ be the derived algebra of $E^{E}$ and let $\mathscr{G}^{\prime}=\mathscr{G} \cap E^{\prime}$. Then $\mathscr{G}=c+\mathfrak{G}^{\prime}$. Since the compact roots of $g$ with respect to $\bar{G}$ can be identified with the roots of $E^{\prime}$ with respect to $\mathscr{G}^{\prime}$, it follows that a linear functional $\Lambda$ on $\zeta^{\prime}$ coincides on $\zeta^{\prime}$ with the highest weight of some irreducible representation of $K^{\prime}$ if and only if $2 \Lambda\left(H_{\alpha}\right) / \alpha\left(H_{\alpha}\right)$ is a nonnegative integer for every compact positive root $\alpha \quad\left(H_{a}\right.$ is the unique element in 5 such that $B\left(H, H_{\alpha}\right)=\alpha(H)$ for all $H \in \mathfrak{K} ; B$ denotes the Killing form on $\left.g \times g\right)$. Therefore the irreducible representations of $K$ are in one-to-one correspondence $\Lambda \leftrightarrow \tau_{\Lambda}$ with the linear functionals $\Lambda$ on $\mathscr{G}$ such that $2 \Lambda\left(H_{\alpha}\right) / \alpha\left(H_{\alpha}\right)$ is a nonnegative integer for every compact positive root $a$.

Let $a_{1}, \cdots, a_{r}$ be the set of all simple roots of $g$; here we are referring to the set $P$ of all positive roots introduced before. Assume that $a_{1}, \cdots, a_{t}(t \leq$ $r)$ are all the noncompact positive roots among these. Define a linear functional $\Lambda_{0}$ on $\mathfrak{b}$ by

$$
\begin{array}{ll}
\Lambda_{0}\left(H_{a_{i}}\right)=0, & 1 \leq i \leq t, \\
\Lambda_{0}\left(H_{a_{i}}\right)=\Lambda\left(H_{a_{i}}\right), & t<i \leq r .
\end{array}
$$

Now, there exists a finite dimensional irreducible representation $\tau$ of $G_{c}$ with highest weight $\Lambda_{0}$. Let $v$ be a nonzero highest weight vector. Let $V$ denote the linear span of $\left\{r(k) v: k \in K_{c}\right\} ; V$ is a $K_{c}$-invariant subspace. Let ${ }^{\tau} \boldsymbol{\Delta}_{0}(k)=\tau(k) \mid V$, for all $k \in K_{c}$, then $\left(V, \tau_{\Lambda_{0}}\right)$ is an irreducible $K_{c}$-module with highest weight $\Lambda_{0}$. Let $\tau_{A}$ be the representation of $K$ on $V$ defined by

$$
\tau_{\Lambda}(k)=e^{\lambda(H)} \tau_{\Lambda_{0}}(\bar{k}), \quad k \in(\exp H) K^{\prime}, H \in c_{0},
$$

where $\lambda=\Lambda-\Lambda_{0}$. We claim that ${ }^{\tau_{\Lambda}}$ is an irreducible representation of $K$ on $V$ with highest weight $\Lambda$. In fact, it is an irreducible representation, because $\tau_{\Lambda_{0}}$ is irreducible, and, if $H \in \mathrm{C}_{0}$,

$$
\dot{\tau}_{\Lambda}(H)=\left[d / d t e e^{\lambda(t H)} \tau_{\Lambda_{0}}\left(\exp _{K_{0}} t H\right)\right]_{t=0}=\lambda(H)+\dot{\tau}_{\Lambda_{0}}(H),
$$

if $X \in \mathfrak{E}_{0}^{\prime}$,

$$
\dot{\tau}_{\Lambda}(X)=\left[d / d t \tau_{\Lambda_{0}}\left(\exp _{K_{0}} t X\right)\right]_{t=0}=\dot{\tau}_{\Lambda_{0}}(X) .
$$


Therefore

$$
\begin{aligned}
& \dot{\tau}_{\Delta}(H) v=\left(\lambda+\Lambda_{0}\right)(H) v=\Lambda(H) v \text { if } H \in c_{0}, \\
& \tau_{\Delta}(H)_{v}=\Lambda_{0}(H)_{v}=\Lambda(H)_{v} \quad \text { if } H \in \mathfrak{G}_{0}^{\circ}=\mathfrak{G}_{0} \cap \mathfrak{E}_{0}^{\prime},
\end{aligned}
$$

hence, the highest weight of $\tau_{A}$ is $\Lambda$.

We recall that we have defined the map $\kappa: G \rightarrow K_{c}$ by

$$
\bar{g}=q \kappa(g) p, \quad q \in P_{-}, \kappa(g) \in K_{c}, p \in P_{+} .
$$

Let $\widetilde{K}_{c}$ denote the universal covering group of $K_{c}$. Since $G$ is simply connected the map $K$ can be lifted, in a unique way, to a map $\tilde{K}: G \rightarrow \widetilde{K}_{c}$ such that $\tilde{K}(e)=$ $e$ and making the following diagram commutative:

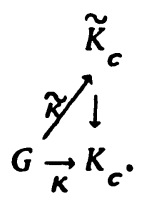

We have the direct decomposition $K^{\prime}=C+E^{\prime}$, hence, if $\tilde{K}_{c}^{\prime}$ and $\tilde{C}$ denote the analytic subgroups of $\widetilde{K}_{c}$ which correspond to the subalgebras $\mathfrak{E}^{\prime}$ and $c$ respectively we have that $\widetilde{K}_{c}=\widetilde{C} \times \widetilde{K}_{c}^{\prime}$ (direct product). Let us define a map $\gamma: G \rightarrow c$ by

$$
\tilde{K}(g) \in \exp (\gamma(g)) \tilde{K}_{c}^{\prime}, \quad \text { for all } g \in G
$$

Now we are in a position to define our desired map $\Phi: G \rightarrow G l(V)$. Let

$$
\Phi(g)=e^{\lambda(\gamma(g))_{r_{0}}}(\kappa(g)), g \in G .
$$

We want to point out the following properties of the map $\Phi$.

2.3. Theorem. (i) $\Phi(e)=I$ identity transformation of $V$.

(ii) $\Phi\left(k_{1} g k_{2}\right)=r_{A}\left(k_{1}\right) \Phi(g) r_{A}\left(k_{2}\right)$ for all $g \in G ; k_{1}, k_{2} \in K$.

(iii) $X \Phi=0$ for all $X \in \mathfrak{P}_{+}$.

Moreover any continuously differentiable function $\Phi_{1}: G \rightarrow L(V)$ wbicb satisfies properties (i), (ii) and (iii) coincides witb the function $\Phi$. lemma.

Before giving the proof of Theorem 2.3 we will state and prove the following

2.4. Lemma. (i) $\kappa\left(k_{1} g k_{2}\right)=\bar{k}_{1} \kappa(g) \bar{k}_{2}$ for all $g \in G ; k_{1}, k_{2} \in K$.

(ii) $d \kappa_{g}(X)=0$ for all $g \in G ; X \in p_{+}$. write

Proof. For $g \in G$, let $\bar{g}=q k(g) p$ where $q \in P_{-}, p \in P_{+}$. Given $k_{1}, k_{2} \in K$, 


$$
\overline{k_{1} g k_{2}}=\bar{k}_{1} \bar{g} \bar{k}_{2}=\bar{k}_{1} q \bar{k}_{1}^{-1} \bar{k}_{1} \kappa(g) \bar{k}_{2} \bar{k}_{2}^{-1} p \bar{k}_{2} .
$$

Since $k P_{ \pm} k^{-1}=P_{ \pm}$for all $k \in K_{c}$, we conclude that $\kappa\left(k_{1} g k_{2}\right)=\bar{k}_{1} \kappa(g) \bar{k}_{2}$. To prove (ii) it is convenient to introduce the following notation: let $\alpha: G \rightarrow G_{c}$ be the map defined by $a(g)=\bar{g}$ and let $\beta: P_{-} K_{c} P_{+} \rightarrow K_{c}$ be the map defined by $\beta(q k p)=k$ whenever $q \in P_{-}, k \in K_{c}$ and $p \in P_{+}$. Suppose $X_{1}, X_{2} \in g_{0}$, then

Now

$$
\begin{aligned}
d \alpha_{B}\left(X_{1}+i X_{2}\right) & =\left[d / d t \alpha\left(g \exp t X_{1}\right)\right]_{t=0}+i\left[d / d t \alpha\left(g \exp t X_{2}\right)\right]_{t=0} \\
& =\left[d / d t\left(\alpha(g) \exp _{G_{c}} t X_{1}\right)\right]_{t=0}+i\left[d / d t\left(\alpha(g) \exp G_{c} t X_{2}\right)\right]_{t=0} \\
& =\left[d / d t\left(\alpha(g) \exp _{G_{c}} t\left(X_{1}+i X_{2}\right)\right)\right]_{t=0}
\end{aligned}
$$

If $X_{1}+i X_{2} \in p_{+}$then

$$
\begin{aligned}
d \kappa_{8}\left(X_{1}+i X_{2}\right) & =d \beta d a_{g}\left(X_{1}+i X_{2}\right) \\
& =d \beta\left[d / d t\left(\alpha(g) \exp _{G_{c}} t\left(X_{1}+i X_{2}\right)\right)\right]_{t=0} \\
& =\left[d / d t\left(\beta\left(\alpha(g) \exp _{G_{c}} t\left(X_{1}+i X_{2}\right)\right)\right)\right]_{t=0} .
\end{aligned}
$$

$$
\beta\left(\alpha(g) \exp _{G_{c}} t\left(X_{1}+i X_{2}\right)\right)=\beta\left(q k(g) p \exp _{G_{c}}\left(X_{1}+i X_{2}\right)\right)=\kappa(g),
$$

since $P_{+}$is a group, and therefore $d \kappa_{g}\left(x_{1}+i X_{2}\right)=0$.

Proof of Theorem 2.3. From part (i) of Lemma 2.4 and the uniqueness of path lifting it follows that $\widetilde{k}\left(k_{1} g k_{2}\right)=\widetilde{k}\left(k_{1}\right) \widetilde{K}(g) \widetilde{K}\left(k_{2}\right)$ for all $g \in G ; k_{1}, k_{2} \in K$; which in turns implies that

$$
\gamma\left(k_{1} g k_{2}\right)=\gamma\left(k_{1}\right)+\gamma(g)+\gamma\left(k_{2}\right) \text { for all } g \in G ; k_{1}, k_{2} \in K \text {. }
$$

Also, we need to observe that if $k=(\exp H) k^{\prime}, H \in c_{0}$ and $k^{\prime} \in K^{\prime}$, then the uniqueness of path lifting implies that $\widetilde{k}(k)=\widetilde{k}(\exp H) \widetilde{k}\left(k^{\prime}\right)$, and moreover that $\widetilde{k}\left(k^{\prime}\right) \epsilon K_{c}^{\prime}$ and $\widetilde{k}(\exp H)=\exp _{\widetilde{K}_{c}}(H)$. Hence $\gamma(k)=H$ if $k \in(\exp H) K^{\prime}, H \in c_{0}$.

Therefore, going back to the definition (2.6) and using part (i) of Lemma 2.4 again, we have

$$
\begin{aligned}
\Phi\left(k_{1} g k_{2}\right) & =e^{\lambda\left(\gamma\left(k_{1} g k_{2}\right)\right)} \tau_{\tau_{0}}\left(k\left(k_{1} g k_{2}\right)\right) \\
& =e^{\lambda\left(\gamma\left(k_{1}\right)\right)} \tau_{\tau_{0}}\left(\bar{k}_{1}\right) \Phi(g) e^{\lambda\left(\gamma\left(k_{2}\right)\right)} \tau_{\tau_{0}}\left(\bar{k}_{2}\right) \\
& =e^{\lambda\left(H_{1}\right)} \tau_{\Delta_{0}}\left(k_{1}\right) \Phi(g) e^{\lambda\left(H_{2}\right)} \tau_{\Delta_{0}}\left(\bar{k}_{2}\right)
\end{aligned}
$$

if $k_{j} \in\left(\exp H_{j}\right) K^{\prime}, H_{j} \in \mathrm{C}_{0}, j=1,2$. By taking a look at (2.5) we realize that part (ii) of the theorem is proved. We will prove now that $X \Phi=0$ for all $X \in \mathfrak{p}_{t}$. Let $\rho: \tilde{K}_{c} \rightarrow K_{c}$ denote the covering projection. Then 


$$
d \rho\left(d \widetilde{\kappa}_{\boldsymbol{g}}(X)\right)=d \kappa_{\boldsymbol{g}}(X)=0 \text { for all } g \in G, X \in \mathfrak{p}_{+} .
$$

Since $d \rho$ is an isomorphism, it follows that $d \widetilde{\kappa}_{g}(X)=0$ for all $X \in p_{+}$. Let $\delta$ : $\widetilde{K}_{c} \rightarrow c$ denote the map defined by $\beta((\exp H) k)=H\left(H \in c, k \in \widetilde{K}_{c}^{\prime}\right)$. Then

$$
d \gamma_{\boldsymbol{g}}(X)=d \beta\left(d \tilde{\kappa}_{\boldsymbol{g}}(X)\right)=0, \text { for all } g \in G, X \in p_{+} \text {. }
$$

Therefore,

$$
\left.[X \Phi](g)=\lambda\left(d \gamma_{g}(X)\right) \Phi(g)+e^{\lambda(\gamma(g))}\left(d \tau_{\Lambda_{0}}\right) \kappa_{(g}\right) d \kappa_{g}(X)=0
$$

for all $g \in G, X \in p_{+}$.

Finally, we have to prove the uniqueness of the map $\Phi$. Let $\Phi_{1}: G \rightarrow L(V)$ $(L(V)$ denotes the space of all linear maps of $V$ into $V$ ) be any continuously differentiable map with the properties (i), (ii) and (iii) of the Theorem 2.3. Then the formula

$$
F(\psi(g K))=\phi_{1}(g) \Phi(g)^{-1}
$$

defines a continuously differentiable map $F: D \rightarrow L(V)$ such that

$$
F(k \cdot z)=\tau_{\mathbf{\Lambda}}(k) F(z) \tau_{\mathbf{\Lambda}}(k)^{-1} \text { for all } k \in K ; z \in D .
$$

In (2.2) we have identified $p_{-}$(resp. $p_{+}$) with the holomorphic (resp. antiholomorphic) tangent space of $D$ at 0 , via the canonical projection $\pi: G \rightarrow D$. Then, since the holomorphic structure of $D$ is $G$-invariant, we have $d \pi_{g} X_{g} \in T_{\pi(g)}$ (resp. $\left.d \pi_{\boldsymbol{g}} X_{\boldsymbol{g}} \in \bar{T}_{\pi(g)}\right)$ if $X \in p_{-}$(resp. $X \in p_{+}$). Thus a continuously differentiable vector valued function on $D$ is holomorphic if and only if $\left(d \pi_{g} X_{g}\right) f=0$ for all $g \in G, X \in p_{+}$.

From (2.7) it follows that

$$
\left(d \pi_{g} X_{g}\right) F=\left[X \Phi_{1}\right](g) \Phi(g)^{-1}-\Phi_{1}(g) \Phi(g)^{-1}[X \Phi](g) \Phi^{-1}(g)
$$

which shows that $F$ is holomorphic since $X \Phi=X \Phi_{1}=0$ for all $X \in p_{+}$.

Let us point out that the action of $K$ on $D$ is given by

$$
k \cdot z=\operatorname{Ad}(k) z \quad \text { for all } k \in K, z \in D \text {. }
$$

Let $C_{0}$ be the analytic subgroup of $K_{0}$ with Lie algebra $C_{0}$. Then $C_{0}$ is a torus. If we decompose $P_{-}$into irreducible submodules under the action of $C_{0}$, the characters which thus appear are nontrivial, since they correspond to the noncompact simple roots which have a nonzero restriction to $c_{0}$. Therefore, if $f$ is a holomorphic vector valued function on $D$ then the Cauchy integral formula tells us that

$$
f(0)=\int_{C_{0}} f(\operatorname{Ad}(b) z) d b \quad \text { for all } z \in D
$$


where $d b$ denotes the normalized Haar measure of $C_{0}$.

We apply (2.9) to the holomorphic function $F$ defined in (2.7):

$$
F(0)=\int_{C_{0}} F(\operatorname{Ad}(b) z) d b=\int_{C_{0}} F(z) d b=F(z) \text { for all } z \in D ;
$$

here we have used (2.8) and the fact that $\tau_{\Lambda}(k)$ is a scalar multiple of the identity for every $k$ in the center of $K$. Thus we have proved that $F(z)=F(0)=\Phi_{1}(e) \Phi(e)^{-1}$ $=I$ (identity of $\mathrm{Gl}(V)$ ) for all $z \in D$ and therefore $\Phi_{1}(g)=\Phi(g)$ for all $g \in G$. This completes the proof of the Theorem 2.3.

The map $\Phi: G \rightarrow G l(V)$ defined in (2.6), and since property (ii) of Theorem 2.3 holds, certainly defines a $C^{\infty}$-equivalence $\Psi$ (see 2.4 ) between the homogeneous holomorphic vector bundle $E_{\Lambda} \rightarrow G / K$ induced by the irreducible representation $\tau_{\Lambda}$ of $K$ on $V$, and the trivial vector bundle $D \times V \rightarrow D$. Therefore, the pair $(\psi, \Psi)$ induced a bijection $s \leftrightarrow s^{\prime}$ between $C^{\infty}$-sections of $E_{\mathbf{\Lambda}} \rightarrow G / K$ and those of $D \times V \rightarrow D$. If we define the functions $f_{s}: G / K \rightarrow V$ and $f_{s^{\prime}}: D \rightarrow V$ by

$$
s(g K)=\left[g, f_{s}(g)\right] \text { and } s^{\prime}(z)=\left(z, f_{s^{\prime}}(z)\right) \text { for all } g \in G, z \in D
$$

then $f_{s}$ and $f_{s^{\prime}}$ are related by

$$
f_{s^{\prime}}(\psi(g K))=\Phi(g) f_{s}(g) \quad \text { for } s \leftrightarrow s^{\prime} \text { and all } g \in G .
$$

According to Theorem 2.1 a $C^{\infty}$-section $s$ of $E_{\Lambda} \rightarrow G / K$ is holomorphic if and only if $X f_{s}=0$ for all $X \in \eta_{+}$. From (2.10) it follows that

$$
\left(d \pi_{g} X_{g}\right) f_{s^{\prime}}=[X \Phi](g) f_{s}(g)+\Phi(g)\left[X f_{s}\right](g) .
$$

Part (iii) of The orem 2.3 implies now that $\left[X f_{s}\right](g)=0$ if and only if $\left(d \pi_{g} X_{g}\right) f_{s^{\prime}}$ $=0$. In other words, the bijection $s \leftrightarrow s^{\prime}$ restricts to a bijection between the holomorphic sections of $E_{\Lambda} \rightarrow G / K$ and the holomorphic sections of $D \times V \rightarrow D$. Hence $\Psi$ is holomorphic.

As an application of the uniqueness part of Theorem 2.3, we will point out here a functional equation satisfied by the map $\Phi: G \rightarrow \mathrm{Gl}(V)$ defined in (2.6).

Let $\tau_{\Lambda}$ be an irreducible unitary representation of $K$ on $V$ and let $\chi_{\Lambda}: K \rightarrow$ $C$ denote its character. Let $\Phi: G \rightarrow G l(V)$ denote the unique map satisfying properties (i), (ii) and (iii) of Theorem 3.3. Then $\overline{\chi_{\Lambda}(k)} \Phi(x \mathrm{~kg})$ depends only on $\bar{k}$. Let $d \bar{k}$ denote the Haar measure on $K_{0}$ normalized by $\int_{K_{0}} d \bar{k}=1$.

\subsection{Theorem.}

$$
(\operatorname{dim} V) \int_{K_{0}} \overline{\chi_{\mathbf{\Lambda}}(k)} \Phi(x k g) d \bar{k}=\Phi(x) \Phi(g) \quad \text { for all } x, g \in G
$$


Proof. Fix $x \in G$. Let

$$
F(g)=(\operatorname{dim} V)\left(\int_{K_{0}} \overline{\chi_{\Lambda}(k)} \Phi(x k g) d \bar{k}\right) \Phi(g)^{-1} .
$$

Then it is easy to check that

$$
\begin{array}{cl}
F(b g k)=F(g) & \text { for all } g \in G, k \in K, b \in \text { center of } K, \\
X F=0 & \text { for all } X \in p_{+} .
\end{array}
$$

These were the two properties we used in Theorem 2.3 to conclude that $F(g)=$ $F(e)$ for all $g \in G$. Now we compute

$$
\begin{aligned}
F(e) & =(\operatorname{dim} V) \Phi(x) \int_{K_{0}} \overline{\chi_{\Lambda}^{(k)} r_{\Lambda}}(k) d \bar{k} \\
& =(\operatorname{dim} V) \Phi(x) \int_{K_{0}} \overline{\chi_{\Lambda_{0}}(k) r_{\Lambda_{0}}}(k) d k=\Phi(x)
\end{aligned}
$$

(see 2.5), as a consequence of Schur orthogonality relations for the irreducible representation $\tau_{\Lambda_{0}}$ of the compact group $K_{0}$ on $V$. This completes the proof of the theorem.

Let $G$ be a connected Lie group, $K$ a compact subgroup. A spberical function of the pair $(G, K)$ is a complex valued continuous function $f$ on $G$, not identically 0 such that

$$
\int_{K} f(x k g) d k=f(x) f(g)
$$

Spherical functions have the properties (a) $f(e)=1$ and (b) $f\left(k_{1} g k_{2}\right)=f(g)$ for all $g \in G ; k_{1}, k_{2} \in K$. If we recall the properties (i) and (ii) of Theorem 2.3 the connection between $(2.11)$ and $(2.12)$ is transparent.

3. Square integrable holomorphic sections. In this chapter we will be referring to the situation and notation introduced in $\$ 2$. In particular, $M=G / K$ denotes a Hermitian symmetric space of noncompact type, and $G$ denotes the universal covering group of $A_{0}(M)$. $\Lambda$ denotes the highest weight of an irreducible representation $\tau_{\Lambda}$ of $K$ on $V$, and $E_{\Lambda} \rightarrow G / K$ denotes the homogeneous holomorphic vector bundle over $G / K$ induced by $\tau_{\Lambda}$ (cf. The orem 2.1 ).

To define a $G$-invariant Hermitian structure on $E_{\Lambda} \rightarrow G / K$ it is necessary and sufficient to have a $K$-invariant inner product on $V$. The one-to-one correspondence between these structures is given by

$$
\left([g, v],\left[g, v^{\prime}\right]\right)=\left(\nu, v^{\prime}\right) \text { for all }[g, v],\left[g, v^{\prime}\right] \in E_{\mathbf{A}}
$$

For an irreducible representation $\tau_{\Lambda}$ of $K$ on $V$ we can define a $K$-invariant inner product on $V$ if and only if $\Lambda$ is a real linear functional on $\zeta$, i.e. $\Lambda\left(H_{a}\right)$ is a real number for all $a \in \Delta$. Thus, from now on $\Lambda$ will denote a real linear 
functional on $\bar{G}$ such that $2 \Lambda\left(H_{\alpha}\right) / \alpha\left(H_{\alpha}\right)$ is a nonnegative integer for every compact positive root $a$. We choose a $K$-invariant inner product $($,$) on V$, which is unique up to a multiplicative constant, and we introduce the corresponding $G$-invariant Hermitian structure on $E_{\Lambda} \rightarrow G / K$ (see 3.1).

Let $H_{\Lambda}$ denote the space of all holomorphic sections $s$ of $E_{\Lambda} \rightarrow G / K$ such that

$$
\int_{G / K}(s(x), s(x)) d x<\infty,
$$

$d x$ denotes the volume element of the Hermitian symmetric space $G / K$. It is not difficult to show that $H_{\Lambda}$ is a Hilbert space with the norm (3.2). Moreover the natural action of $G$ on the sections of $E_{\Lambda} \rightarrow G / K$, given by

$$
(g s)(x)=g s\left(g^{-1} x\right), \quad g \in G, x \in G / K,
$$

defines a unitary representation $\pi_{\Lambda}$ of $G$ on $H_{\Lambda}$.

By making use of the holomorphic trivialization (2.3) defined by (2.4) and (2.6) the space $H_{\mathrm{A}}$ can be identified with the space of all holomorphic functions $f: D \rightarrow V$ such that

$$
j_{D}(f(z), f(z))_{z} d z<\infty
$$

where $(v, w)_{z}=\left(\Phi(g)^{-1} v, \Phi(g)^{-1} w\right), z=g \cdot 0, v, w \in V$, and $d z$ is the volume element of $D$ corresponding to $d x$ under the map $\psi$. The action of $G$ is given by

$$
(u f)(g \cdot 0)=\Phi(g) \Phi\left(u^{-1} g\right)^{-1} f\left(u^{-1} g \cdot 0\right), \quad u, g \in G,
$$

and in particular for $k \in K$ we have

$$
(k f)(z)=\tau_{\Delta}(k) f\left(\operatorname{Ad}(k)^{-1} z\right), \quad z \in D,
$$

since the action of $K$ on $D$ is given by the adjoint representation of $K$ on $P_{-}$ restricted to $D$; see also Theorem 2.3.

3.1. Theorem (cf. Theorem 2 of [2]). The representation $\pi_{\Lambda}$ of $G$ on $H_{A}$ is irreducible. Moreover, $H_{\Lambda} \neq 1\{0\}$ if and only if $H_{\Lambda}$ contains the constant functions.

Proof. Let $H$ be a nonzero closed invariant subspace of $H_{A}$. Take a function $f \in H$ such that $f(0) \neq 0$. For a fixed $b \in C_{0}$ the formula (3.4) shows that the function $z \rightarrow f(\operatorname{Ad}(b) z)$ is also in $H$, and therefore the constant function $z$ $\rightarrow f(0)$ belongs to $H$ (see 2.9). Thus any two nonzero closed invariant subspaces of $H_{\Lambda}$ have a nonzero intersection. Since $\pi_{\Lambda}$ is unitary, the theorem is completely proved.

The orem 3.1 leads us to consider the integral 


$$
\int_{D}(v, v)_{z} d z=\int_{G_{0}}\left(\Phi(g)^{-1} v, \Phi(g)^{-1} v\right) d \bar{g}, \quad v \in V ;
$$

note that $\left(\Phi(g)^{-1} v, \Phi(g)^{-1} v\right)$ depends only on $\bar{g}$. To compute this, one transforms the integration over $G_{0}$ as follows. (Cf. [7].) Let $a_{p_{0}}$ be a maximal abelian subspace of $p_{0}$ and extend $a_{p_{0}}$ to a maximal abelian subalgebra $a_{0}$ of $g_{0}$. Let $a$ be the subalgebra of $g$ generated by $a_{0}$. Then $a$ is a Cartan subalgebra of $g$ and let $a^{*}=\Sigma R H_{a}$ where $a$ runs over the set of all nonzero roots of $g$ with respect to $a$. Then $a_{p_{0}} \subset a^{*}$ and compatible orderings can be introduced in the dual spaces of $a_{p_{0}}$ and $a^{*}$. Let $Q$ denote the set of positive roots which do not vanish identically on $a_{p_{0}}$. The Killing form of $g_{0}$ gives tise to a Euclidean metric on $a_{p_{0}}$. Let $d H$ denote the Riemannian measure on $a_{p_{0}}$ induced by this metric. Then if $f$ is a continuous function with compact support on $G_{0}$

$$
\int_{G_{0}} f(y) d y=\int_{a_{p_{0}}}\left|\prod_{a \in Q} \sinh \alpha(H)\right| d H \int_{K_{0} \times K_{0}} f\left(k_{1} \exp H k_{2}\right) d k_{1} d k_{.2}
$$

( $d y$ and $d k$ suitable normalized Haar measures).

In the special situation here, it is possible to select $a_{p_{0}}$ with particular reference to $\Delta$. In fact one can find noncompact positive roots $\gamma_{1}, \cdots, \gamma_{s}$ such that $\gamma_{i} \pm \gamma_{j}$ is never a root for $i \neq 1 j$ and the real subspace $a_{p_{0}}$ generated by $X_{\gamma_{i}}+X_{-\gamma_{i}}$ is a maximal abelian subspace of $p_{0}$ (see [7]).

In order to reduce the problem of computing (3.5) to an integral computed in [4], we will consider the homogeneous holomorphic vector bundle $E_{\Lambda *} \rightarrow G / K$ associated to the contragredient representation of ${ }^{\tau_{\Lambda}}$ instead of $E_{\Lambda} \rightarrow G / K$.

Let $V^{*}$ denote the dual space of $V$. The map $v \rightarrow 1_{v}$ defined by $1_{v}(w)=$ $(w, v)$ defines a real isomorphism of $V$ onto $V^{*}$. We can define an inner product on $V^{*}$ by

$$
\left(1_{v}, 1_{w}\right)=(w, v), \quad v, w \in V
$$

Given a linear transformation $T: V \rightarrow V$ then $T^{*}: V \rightarrow V$ will denote the adjoint of $T$ and ${ }^{t} T: V^{*} \rightarrow V^{*}$ will denote the transpose transformation. One can easily check that ${ }^{t} T 1_{v}=1_{T^{*}}$. The map $\Phi^{*}: G \rightarrow \mathrm{Gl}\left(V^{*}\right)$ corresponding to the contragredient representation of $\tau_{\Lambda}$ (se e 2.6 ) is given by

$$
\Phi^{*}(g)=e^{-\lambda(\gamma(g)) t} \tau_{\Lambda_{0}}(k(g))^{-1}, \quad g \in G,
$$

as one can be convinced by using the uniqueness part of Theorem 2.3.

The function we now want to integrate over $G_{0}$ is 


$$
\begin{aligned}
\left(\Phi^{*}(g)^{-1} 1_{v}, \Phi^{*}(g)^{-1} 1_{v}\right) & =\left|e^{\lambda(\gamma(g))}\right|^{2}\left({ }^{t} \tau_{\Lambda_{0}}(\kappa(g)) 1_{v},{ }^{t} \tau_{\Lambda_{0}}(\kappa(g)) 1_{v}\right) \\
& =\left|e^{\lambda(\gamma(g))}\right|^{2}\left({ }^{1} \tau_{\Lambda_{0}}(\kappa(g))^{*} v,{ }^{1} \tau_{\Lambda_{0}}(\kappa(g))^{*} v\right) \\
& =\left|e^{\lambda(\gamma(g))}\right|^{2}\left(\tau_{\Lambda_{0}}(\kappa(g))^{*} v, \tau_{\Lambda_{0}}(\kappa(g))^{*} v\right), \quad g \in G .
\end{aligned}
$$

For $k_{1}, k_{2} \in K$ and $H \in a_{p_{0}}$ we have

$$
\begin{aligned}
& \left(\Phi^{*}\left(k_{1} \exp H k_{2}\right)^{-1} 1_{v}, \Phi^{*}\left(k_{1} \exp H k_{2}\right)^{-1} 1_{v}\right) \\
& =\left|e^{\lambda(\gamma(\exp H))}\right|^{2}\left(\tau_{\Lambda_{0}}(\kappa(\exp H))^{*} \tau_{\Lambda_{0}}\left(\bar{k}_{1}^{-1}\right) \nu, \tau_{\Lambda_{0}}(\kappa(\exp H))^{*} \tau_{\Lambda_{0}}\left(\bar{k}_{1}^{-1}\right) v\right) \\
& =e^{2 \lambda(\gamma(\exp H))}\left\|_{\tau_{\Lambda_{0}}}(\kappa(\exp H)) \tau_{\Lambda_{0}}\left(\bar{k}_{1}^{-1}\right) \nu\right\|^{2}
\end{aligned}
$$

where we have taken into account the fact that $\lambda(\gamma(\exp H))$ is real and that ${ }^{\tau_{\Lambda_{0}}}(\kappa(\exp H))$ is selfadjoint for all $H \in a_{p_{0}}$.

According to (3.6) we have to compute

$$
\int_{K_{0}}\left\|\tau_{\Lambda_{0}}(\kappa(\exp H))_{\tau_{\Lambda_{0}}}(k) \nu\right\|^{2} d k=(\operatorname{dim} V)^{-1} \chi_{\Lambda_{0}}\left(\kappa(\exp H)^{2}\right)
$$

if $\|v\|=1$, where $\chi_{\Delta_{0}}$ denotes the character of ${ }^{\tau_{\Lambda_{0}}}$. This is a straightforward consequence of the Schur orthogonality relations for the irreducible representation $r_{\Delta_{0}}$ of the compact group $K_{0}$ on $V$.

Thus the problem of computing

$$
\int_{D}\left(1_{v}, 1_{v}\right) d z
$$

has been reduced to the more concrete problem of evaluating

$$
\int_{a_{p_{0}}} e^{2 \lambda(\gamma(\exp H))} \chi_{\Lambda_{0}}\left(\kappa(\exp H)^{2}\right) \prod_{a \in Q}|\sinh \alpha(H)| d H .
$$

Let $f_{v^{\prime}}$ denote the constant function $f(z)=v^{\prime}, z \in D, v^{\prime} \in V^{*},\left\|v^{\prime}\right\|=1$ and let $\rho$ denote the half sum of all positive roots of $g$ with respect to $\xi_{\text {. }} H_{\Delta^{*}}$ will denote the space of all square integrable holomorphic sections of $E_{\Lambda^{*}} \rightarrow G / K$ (see 3.2).

3.2. Theorem (cf. Theorem 4, [4]). In order to bave $H_{\Lambda^{*}} \neq\{0\}$ it is neces. sary and sufficient that $\Lambda\left(H_{\beta}\right)+\rho\left(H_{\beta}\right)<0$ for all noncompact positive roots $\beta$. Moreover, it is possible to normalize the Haar measure on $G_{0}$ in such a way that (when $H_{\mathbf{A}} \neq\{0\}$ )

$$
\begin{aligned}
\left\|f_{v^{\prime}}\right\|^{2} & =\int_{G_{0}}\left\|\Phi^{*}(g)^{-1} v^{\prime}\right\|^{2} d \bar{g} \\
& =(\operatorname{dim} V)\left|\prod_{a \in P}\left(\Lambda\left(H_{a}\right)+\rho\left(H_{a}\right)\right) / \rho\left(H_{a}\right)\right|^{-1} .
\end{aligned}
$$


(We recall that $P$ denotes the set of all positive roots of 9 with respect to b.)

3.3. Remark . Let $v_{0} \neq 0$ be a highest weight vector of the irreducible representation $\tau_{\Lambda}$. From (3.3) and (3.4) one easily deduces that

$$
\pi_{\Delta}\left(X_{a}\right) f_{v_{0}}=0 \text { and } \pi_{\Delta}\left(H_{a}\right) f_{v_{0}}=\Lambda\left(H_{\alpha}\right) f_{v_{0}}
$$

for all positive roots $a$ of $g$ with respect to $h$. That is to say that $\pi_{\Delta}$ has an extreme vector $f_{\nu_{0}}$ of weight $\Lambda$. Conversely, the representations $\pi_{\Delta}$ constructed here are precisely all the irreducible un itary representations of $G$ which admit an extreme vector.

3.4. Remark. If we realized $G / K$ as a bounded domain in $p_{+}$instead of in $P_{-}$then we do not need to pass to the contragredient representation of $\tau_{A}$, and the statement of Theorem 3.2 is still correct by replacing the space $H_{\Lambda *}$ by $H_{\Lambda}$.

Square integrable representations. Let $G$ be a connected semisimple Lie group and let $Z$ denote its center. Then if $\pi$ is an irreducible unitary representation of $G$ on a Hilbert space $H$ we can find a unitary character $\eta_{\pi}$ of $Z$ such that $\pi(z)=\eta_{\pi}(z) \pi(e)$ for $z \in Z$. Let $Z_{0}$ be a subgroup of $Z$ of finite index. Let $\boldsymbol{g} \rightarrow \overline{\boldsymbol{g}}$ denote the natural projection of $G$ onto $G_{0}=G / Z_{0}$. Given two nonzero elements $f_{1}$ and $f_{2}$ in $H$, it is clear that $\left|\left(\pi(g) f_{1}, f_{2}\right)\right|(g \in G)$ depends only on $\overline{\boldsymbol{g}}$ and thus defines a continuous function of $G_{0}$. We say that $\pi$ is square integrable, if there exist two elements $f_{1} \neq 0, f_{2} \neq 0$ in $H$ such that

$$
\int_{G_{0}}\left|\left(\pi(g) f_{1}, f_{2}\right)\right|^{2} d \bar{g}<\infty \text {. }
$$

It is obvious that this definition does not depend on the choice of the subgroup $Z_{0}$ as long as $Z / Z_{0}$ is finite.

Let $\pi$ and $\pi^{\prime}$ be two square integrable representations of $G$ on the Hilbert spaces $H$ and $H^{\prime}$ respectively. Then if their central characters coincide on $Z_{0}$ it is clear that $\left(\pi(g) f_{1}, f_{2}\right)\left(\pi^{\prime}(g) f_{1}^{\prime}, f_{2}^{\prime}\right)$ depends only on $\bar{g}$ and therefore may be regarded as a function on $G_{0}\left(f_{1}, f_{2} \in H ; f_{1}^{\prime}, f_{2}^{\prime} \in H^{\prime} ; g \in G\right)$. Then the following Schur orthogonality relations hold (cf. [4]). If $\pi$ and $\pi^{\prime}$ are not equivalent

$$
\int_{G_{0}}\left(\pi(g) f_{1}, f_{2}\right) \overline{\left(\pi^{\prime}(g) f_{1}^{\prime}, f_{2}^{\prime}\right)} d \bar{g}=0
$$

for all $f_{1}, f_{2} \in H$ and $f_{1}^{\prime}, f_{2}^{\prime} \in H^{\prime}$, and

$$
\left.\int_{G_{0}}\left(\pi(g) f_{1}, f_{2}\right) \overline{\left(\pi(g) f_{1}^{\prime}\right.}, \overline{f_{2}^{\prime}}\right) d \bar{g}=d_{\pi}^{-1}\left(f_{1}, f_{1}^{\prime}\right) \overline{\left(f_{2}, f_{2}^{\prime}\right)}
$$

$\left(f_{1}, f_{2}, f_{1}^{\prime}, f_{2}^{\prime} \in H\right)$, where $d_{\pi}$ is a positive constant called the formal degree of $\pi$. Clearly $d_{\pi}$ depends on the choice of $Z_{0}$ and the normalization of the Haar measure of $G_{0}$. However once these have been fixed, it is obvious that two equivalent square integrable representations have the same formal degree. 
Now we are going to prove that the representation $\pi_{\Lambda^{*}}$ of $G$ on $H_{\Lambda^{*}}$ is square integrable and compute its formal degree.

Let us assume that $H_{A} \neq\{0\}$. Then according to the Theorem 3.1, for any $v \in V$ the constant function $f_{v} \in H_{\Lambda}$, hence

$$
\int_{G_{0}}\left(\Phi(x g)^{-1} v, \Phi(g)^{-1} w\right) d \bar{g}=\left(\pi_{\Delta}\left(x^{-1}\right) f_{v}, f_{w}\right)<\infty \quad \text { all } x \in G ; v, w \in V
$$

(see 3.3). Therefore, $\left(\Phi(g)^{-1}\right) * \Phi(x g)^{-1}$ as a function of $\bar{g}$ is integrable over $G_{0}$ for all $x \in G$. Let

$$
\tilde{F}(x)=\Phi(x) \int_{G_{0}}\left(\Phi(g)^{-1}\right)^{*} \Phi(x g)^{-1} d \bar{g}
$$

One can easily check that

(i) $\tilde{F}\left(k_{1} x k_{2}\right)=\tau_{\Delta}\left(k_{1}\right) \widetilde{F}(x) \tau_{\Delta}\left(k_{1}\right)^{-1}$ for all $x \in G ; k_{1}, k_{2} \in K$.

(ii) $X \widetilde{F}=0$ for all $X \in p_{+}$

(cf. Theorem 3.3). Thus $\widetilde{F}$ defines a holomorphic function $F$ on $D$, namely

$$
F(\psi(g K))=\tilde{F}(g) \text { all } g \in G \text {. }
$$

The Cauchy's integral formula (2.9) and property (i) give

$$
F(0)=\int_{C_{0}} F(\operatorname{Ad}(b) z) d b=F(z) \text { for all } z \in D ;
$$

we have taken into account the fact that $\tau_{\Delta}(k)$ is a scalar whenever $k$ is in the center of $K$. Therefore, $\widetilde{F}(x)$ is equal to a constant. To evaluate this constant $\widetilde{F}(e)$ we observe that

$$
\tilde{F}(e)=\tau_{\Delta}(k) \tilde{F}(e) \tau_{\Lambda}(k)^{-1} \text { for all } k \in K
$$

(cf. (i)). Hence $\widetilde{F}(e)$ is a scalar. To evaluate the scalar we just take a vector $v \in V$ of unit length and compute

We have proved

$$
\tilde{F}(e) v, v)=\int_{G_{0}}\left(\Phi(g)^{-1} v, \Phi(g)^{-1} v\right) d \bar{g}=\left\|f_{v}\right\|^{2} .
$$

3.5. Lemma. If the space $H_{\Delta} \neq\{0\}$ and $v \in V$ is such that $\|v\|=1$ then

$$
\int_{G_{0}}\left(\Phi(g)^{-1}\right)^{*} \Phi(x g)^{-1} d \bar{g}=\left\|f_{\nu}\right\|^{2} \Phi(x)^{-1} \quad \text { for all } x \in G \text {. }
$$

We now consider the space $H_{\Lambda *}$ associated with the contragredient representation of $r_{A}$ and we assume that $H_{A^{*}} \neq\{0\}$. To prove that the representation $\pi_{A^{*}}$ of $G$ is square integrable we will consider

$$
\int_{G_{0}}\left|\left(\pi_{\Delta^{*}}(x) f_{v^{\prime}}, f_{v^{\prime}}\right)\right|^{2} d \bar{x}, \quad v^{\prime} \in V^{*},\left\|v^{\prime}\right\|=1
$$


According to Lemma 3.5 we have

$$
\begin{aligned}
\left(\pi_{\mathbf{A}^{*}}(x) f_{v^{\prime}}, f_{v^{\prime}}\right) & =\int_{G_{0}}\left(\Phi^{*}\left(x^{-1} g\right)^{-1} v^{\prime}, \Phi^{*}(g)^{-1} v^{\prime}\right) d \bar{g} \\
& =\left\|f_{v^{\prime}}\right\|^{2}\left(\Phi^{*}\left(x^{-1}\right)^{-1} v^{\prime}, v^{\prime}\right)
\end{aligned}
$$

hence, the integral $(3.10)$ is equal to

$$
\left\|f_{v^{\prime}}\right\|^{4} \int_{G_{0}}\left|\left(\Phi^{*}(x)^{-1} v^{\prime}, v^{\prime}\right)\right|^{2} d \bar{x}
$$

By a computation similar to the one we did before one obtains that

$$
\begin{aligned}
& \left|\left(\Phi^{*}\left(k_{1} \exp H k_{2}\right)^{-1} v^{\prime}, v^{\prime}\right)\right|^{2} \\
& \quad=e^{2 \lambda(\gamma(\exp H))\left|\left(\tau_{\Lambda_{0}}\left(\bar{k}_{1}\right) \tau_{\Lambda_{0}}(\kappa(\exp H)) \tau_{\Lambda_{0}}\left(\bar{k}_{2}\right) \nu, v\right)\right|^{2}}
\end{aligned}
$$

for all $k_{1} k_{2} \in K ; H \in a_{p_{0}}$ and $\nu^{\prime}=1$. Using the Schur orthogonality relations of $\tau_{\Lambda_{0}}$ on $K_{0}$ it follows that

$$
\begin{aligned}
\int_{K_{0} \times K_{0}}\left|\left(\tau_{\Lambda_{0}}\left(k_{1}\right) \tau_{\Lambda_{0}}(\kappa(\exp H)) \tau_{\Lambda_{0}}\left(k_{2}\right) \nu, v\right)\right|^{2} d k_{1} d k_{2} \\
\quad=(\operatorname{dim} V)^{-1} \int_{K_{0}}\left\|r_{\Lambda_{0}}(\kappa(\exp H)) \tau_{\Lambda_{0}}\left(k_{2}\right) \nu\right\|^{2} d k_{2} \\
=(\operatorname{dim} V)^{-1} \chi_{\Lambda_{0}}\left(\kappa(\exp H)^{2}\right)
\end{aligned}
$$

(see (3.7)). Making use of (3.6), (3.11), (3.12) and (3.13) we obtain

$$
\int_{G_{0}}\left|\left(\pi_{\Lambda^{*}}(x) f_{v^{\prime}}, f_{v^{\prime}}\right)\right|^{2} d x=(\operatorname{dim} v)^{-1}\left\|f_{v^{\prime}}\right\|^{6}<\infty .
$$

Therefore, $\pi_{\Lambda^{*}}$ is a square integrable representation of $G$ and according to (3.8) and (3.9) its formal degree is given by

$$
d_{\pi_{\Lambda^{*}}}=(\operatorname{dim} V)\left\|f_{v^{\prime}}\right\|^{-2}=\left|\prod_{a \in P}\left(\Lambda\left(H_{a}\right)+\rho\left(H_{a}\right)\right) / \rho\left(H_{a}\right)\right| .
$$

Note the similarity of this formula with the formula of Weyl which gives the dimension of an irreducible representation of a compact semisimple Lie group in terms of its highest weight.

3.6. Remark. The Hodge star operator induces a conjugate linear isomorphism between the Hilbert space $H_{\mathbf{\Lambda}}=H_{2}^{0,0}\left(E_{\mathbf{\Lambda}}\right)$ of all square integrable holomorphic sections of $E_{\Lambda} \rightarrow G / K$ and the space $H_{2}^{0, n}\left(L \otimes E_{\Lambda}^{*}\right)$ of all square integrable harmonic $(0, n)$ forms on $G / K$ with values in $L \otimes E_{\Lambda^{*}}^{*} L$ denotes the canonical line bundle of $G / K$ and $E_{\Lambda}^{*}$ denotes the complex dual bundle of $E_{\Lambda}$. Thus the action of $G$ on $H_{2}^{0, n}\left(L \otimes E_{A}^{*}\right)$ defines also a unitary irreducible representation of $G$ which is square integrable over $G / Z$. 


\section{REFERENCES}

1. W. L. Baily and A. Borel, Compactification of arithmetic quotients of bounded symmetric domains, Ann. of Math. (2) 84 (1966), 442-528. MR $35 \# 6870$.

2. F. Bruhat, Travaux de Harish-Chandra, Séminaire Bourbaki, 9ième année: 1956/57, 2nd ed., Exposé 143, Secrétariat mathématique, Paris, 1959, pp. 1-9. MR 28 \#1090.

3. Harish-Chandra, Representations of semisimple Lie groups. V, Amer. J. Math. 78 (1956), 1-41. MR 18, 490.

4. - Representations of semisimple Lie groups. VI. Integrable and square-integrable representations, Amer. J. Math. 78 (1956), 564-628. MR 18, 490.

5. - Discrete series for semisimple Lie groups. I. Construction of invariant eigendistributions, Acta Math. 113 (1965), 241-318. MR 36 \#2744.

6. - Discrete series for semisimple Lie groups. II. Explicit determination of the characters, Acta Math. 116 (1966), 1-111. MR 36 \#2745.

7. S. Helgason, Differential geometry and symmetric spaces, Pure and Appl.. Math., vol. 12, Academic Press, New York, 1962. MR 26 \#2986.

8. S. Kobayashi and K. Nomizu, Foundations of differential geometry. Vol. II, Interscience Tracts in Pure and Appl. Math., no. 15, Interscience, New York, 1969. MR 38 \#6501.

9. M. S. Narasimhan and K. Okamoto, An analogue of the Borel-Weil-Bott theorem for Hermitian symmetric pairs of non-compact type, Ann. of Math. (2) 91 (1970), 486-511. MR 43 \#0419.

10. J. A. Tirao and J. A. Wolf, Homogeneous holomorphic vector bundles, Indiana Univ. Math. J. 20 (1970/71), 15-31. MR 41 \#7715.

DEPARTMENTO DE MATEMÁTICA, UNIVERSIDAD NACIONAL DE CóRDOBA, CóRDOBA, ARGENTINA

Current address: Department of Mathematics, Uni versity of California, Berkeley, California 94720 